\title{
Publisher's Note: Spatial Search by Quantum Walk is Optimal for Almost All Graphs [Phys. Rev. Lett. 116, 100501 (2016)]
}

Shantanav Chakraborty, Leonardo Novo, Andris Ambainis, and Yasser Omar

(Received 26 May 2016; published 13 June 2016)

DOI: 10.1103/PhysRevLett.116.249901

This paper was published online on 11 March 2016 with an omission of two references and a Note. Reference [30] should read as "B. Hein and G. Tanner, Wave Communication across Regular Lattices, Phys. Rev. Lett. 103, 260501 (2009)." Reference [31] should read as "J. Böhm, M. Bellec, F. Mortessagne, U. Kuhl, S. Barkhofen, S. Gehler, H. Stöckmann, I. Foulger, S. Gnutzmann, and G. Tanner, Microwave Experiments Simulating Quantum Search and Directed Transport in Artificial Graphene, Phys. Rev. Lett. 114, 110501 (2015)." A Note was inserted on page 5 that reads as "Note added.- Recently we became aware of Refs. [30, 31], where the spatial search algorithm has been adapted for quantum state transfer in regular lattices." The paper has been corrected as of 31 May 2016. The references and Note are not present in the printed version of the journal. 